\title{
Design of Remote Temperature Monitoring System Based on GPRS
}

\author{
Qiang Qi ${ }^{1, a}$, Bao-hua Jiang ${ }^{2, b,}$, Pei-xue Liu ${ }^{2, c}$ and Li Dong ${ }^{2, d}$ \\ ${ }^{1}$ Binhai University Mechanical and Electrical Engineering College, China \\ ${ }^{2}$ Qingdao Huanghai University Mechanical and Electrical Engineering College, Qingdao, China

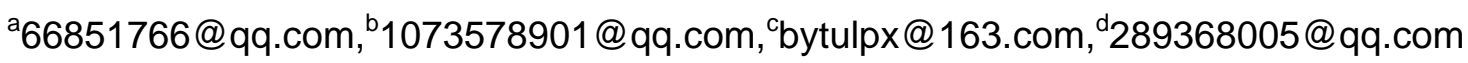

Keywords: Single chip microcomputer, Remote monitoring system, Temperature detection, GPRS.

\begin{abstract}
In this study, a kind of remote temperature monitoring system based on GPRS, used STC89C51 microcontroller is put forward as the host controller design, GPRS network as a remote signal transmission platform. The system can realize the measurement of temperature, also can realize the control of temperature to a certain extent. Using DS18B20 as the temperature detection element, the signal transformation was performed by $\mathrm{A} / \mathrm{D}$, and the system is showed by a high bright 12864 LCD. The testing results showed that the design can show the temperature of each point in time. When there are abnormal conditions, the parameters can be corrected and the number of detection units can be expanded.
\end{abstract}

\section{Introduction}

With the rapid development of network technology and computer technology, the application of computer remote wireless monitoring technology in the field of industrial control has become more and more extensive and has very important significance. GPRS is a new kind of wireless communication between users and data, which is characterized by high speed, high efficiency and low economy. GPRS terminal is designed to attach to GPRS network and send and receive data through GPRS. GPRS network has no blind spots in the world, so as long as there is a signal, GPRS can be connected. Using GPRS to communicate in remote temperature monitoring is of great application scope ${ }^{[1]}$.

In this paper, a remote temperature monitoring system based on GPRS is proposed, which is used to process the temperature data collected by the microcontroller and transmit it to the computer via GPRS. The hardware mainly realizes the temperature data collection and processing, and displays the temperature on the liquid crystal display, the alarm is carried out when the temperature is too high, and finally the remote transmission is carried out ${ }^{[2]}$. The main functions of software design are data acquisition and wireless transmission. In practice, check the tests and solve the problems that arise.

\section{Design of remote temperature monitoring system}

The overall scheme design of the system

This design is based on the design of GPRS remote temperature monitoring system, which is the core of the single-chip microcomputer, so that it can complete the processing of collecting data and then send the results to the computer through GPRS communication. The specific implementation process is: after the physical, the DS18B20 detects the temperature around the 
module and sends it to the MCU, and the LCD will display the temperature value. Then sent the data by the GPRS module, through a fixed IP TCP connection to computer and GPRS module, the data is transferred to upper computer, namely the detected temperature is displayed on the server that builds the server.

According to the function implementation of the lower computer, the hardware circuit of the lower part of the system is designed in modular form.

\section{Communication module design}

GPRS network transmission platform

The layout of the transmission platform of GPRS network is hierarchical and consists of layered protocol structures. It is the transmission of data in each GPRS and the control of this data transfer. It is a transmission platform created by connecting the wireless interface and the NSS platform, and this is done by the Gb interface. The GPRS transport protocol platform is shown in figure 1.

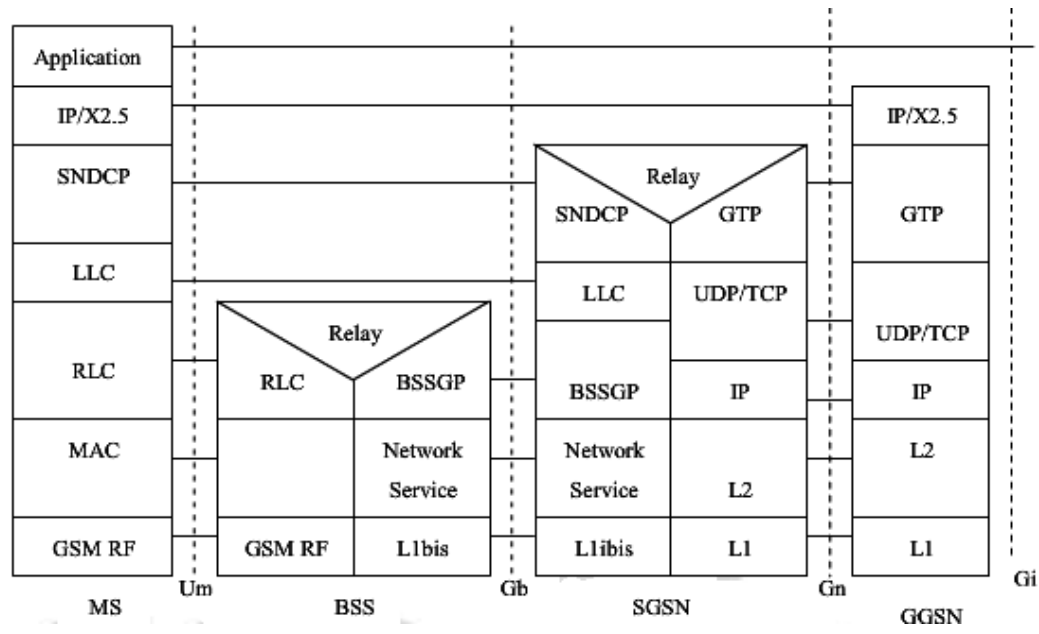

Fig.1 GPRS transport protocol platform

Communication module structure

In this system, the connection between the lower computer and the monitoring center is transmitted through GPRS. The GPRS module here will be the key to realize this link. The module selected here is the goouuu-a6 module (See Fig.2).

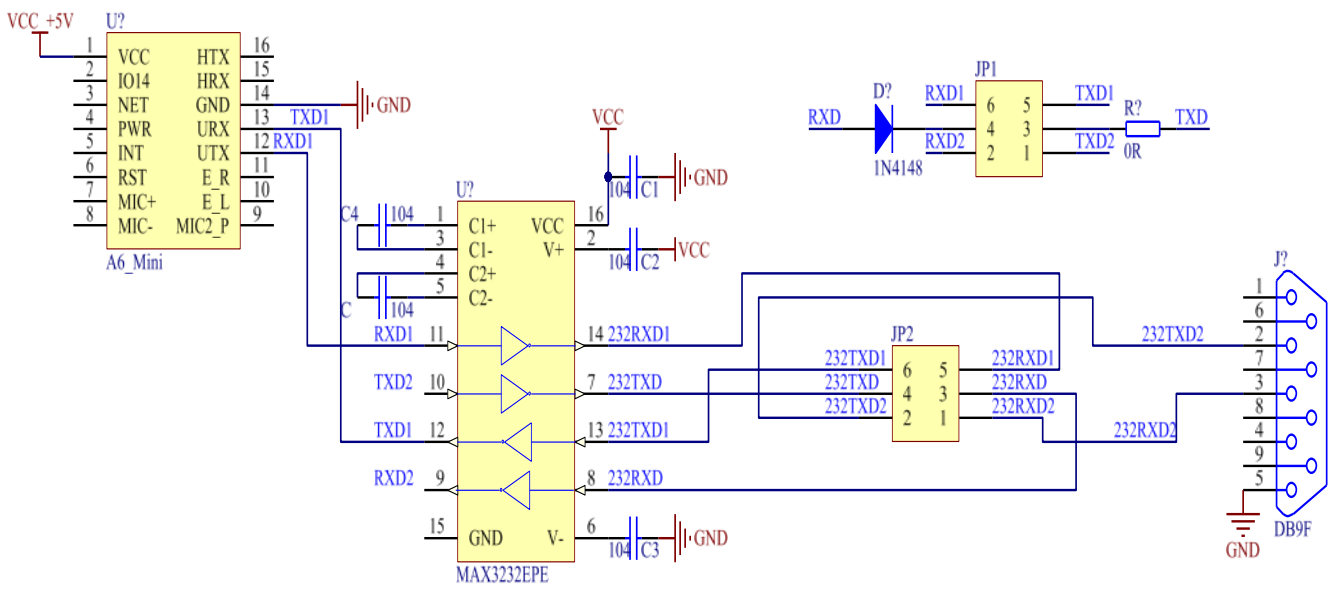

Fig.2 RS232 serial port module schematic diagram

Physical design has RS232 interface, serial port communication. The rs-232 protocol represents logic 1 when the voltage is negative $-5 \sim-15 \mathrm{v}$, which is 0 when the voltage is positive $+5 \sim 15 \mathrm{~V}$. The serial interface chip is MAX3232EPE here, it is a low power to the transmitter, 
communication speed, can reach 1 MBPS, it is ready to work only needs connecte to four capacitance, the power supply voltage of $3 \sim 5.5 \mathrm{~V}$. The circuit diagram of RS232 serial port module is shown in figure 2.

\section{Hardware design of remote temperature monitoring system}

Used STC89C51 series microcontroller, mainly has the single chip microcomputer connection temperature sensors (temperature acquisition and processing), liquid crystal display (display temperature), a buzzer (high temperature alarm) GPRS system (such as remote data transmission $)^{[3]}$.

Single chip microcomputer module

The STC89C51 single chip has 40 pins, and the working voltage is $5.5 \mathrm{~V}-3.3 \mathrm{~V}$, and there are 216 timer/calculagraph, and the chip is integrated with the general 8-bit central processor and Flash storage unit. STC89C51 has a wide application in the electronic production equipment industry ${ }^{[4]}$.

The minimum system consists of single chip microcomputer, crystal oscillator circuit and reset circuit.

Crystal oscillation circuit: crystals take $11.0592 \mathrm{MHZ}$, crystals like the heart of the single chip microcomputer, microcomputer runtime to provide a stable clock precision, ensure the MCU internal and external circuit of the sequential logic circuit coordinated action.

Reset circuit: it needs to be reset either when the MCU is first connected to the power supply or when the fault occurs in the running process. The reset circuit is used to restore the state of each circuit in the single chip to a determined initial value and start working in this state.

Temperature acquisition module

The temperature sensor is the DS18B20 with a voltage range of $3 \sim 5 \mathrm{~V}$ and has its own single line interface, which can complete each other's communication by connecting a single line with a single chip. Temperature range in $-55{ }^{\circ} \mathrm{C} \sim+125{ }^{\circ} \mathrm{C}$, the inherent temperature measuring resolution of $0.5{ }^{\circ} \mathrm{C}$. The measured results were sent in nine digital quantities.

\section{LCD module}

Used LCD12864 LCD, including RS, RW, E serial slices respectively selected data signal, serial port, serial synchronous clock, pin is Shared with other modules, respectively connected to microcontroller P3.7, P3.6, P3.5. PSB and/RST are respectively and/string selection and LCD $\mathrm{P} 3.4$ and P3.3. The schematic diagram of the connecting circuit is shown in figure 3.

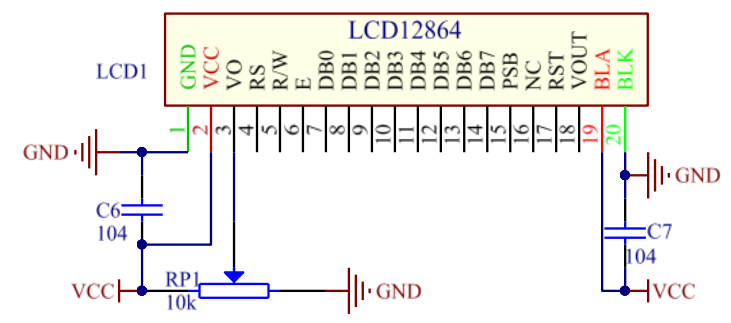

Fig.3 LCD connection schematic diagram

Logic (VDD) : working voltage from $4.5 \mathrm{~V}$ to $5.5 \mathrm{~V}$ power supply ground (GND) : $0 \mathrm{~V}$ working temperature (Ta) : $0 \sim 60{ }^{\circ} \mathrm{C}$ (room temperature) / - 20 $75{ }^{\circ} \mathrm{C}$ temperature (wide).

1.3.4 Sound and light alarm module 
Including sound and light alarm module in this system, when in the monitoring state and temperature is higher than the set value, and P0.5 MCU P0.0 out high level, and the emitter transistor collector conduction, LED lights, the alarm.

\section{Software design of remote temperature monitoring system}

Master programming

The main job of software design is to be able to receive the SCM from the temperature information, and the temperature data are processed and displayed in chronological order, with GPRS module for data transmission, to achieve the purpose of remote temperature monitoring. The main program module mainly let initialized communication module, before that, of course, through a serial port using the AT command set, to the temperature sensor is the temperature for the analysis and processing, finally through the A6 module value AT present on the computer, and then will be continuous loop over the steps ${ }^{[5]}$. Some of the flowcharts are shown in figure 4 below.

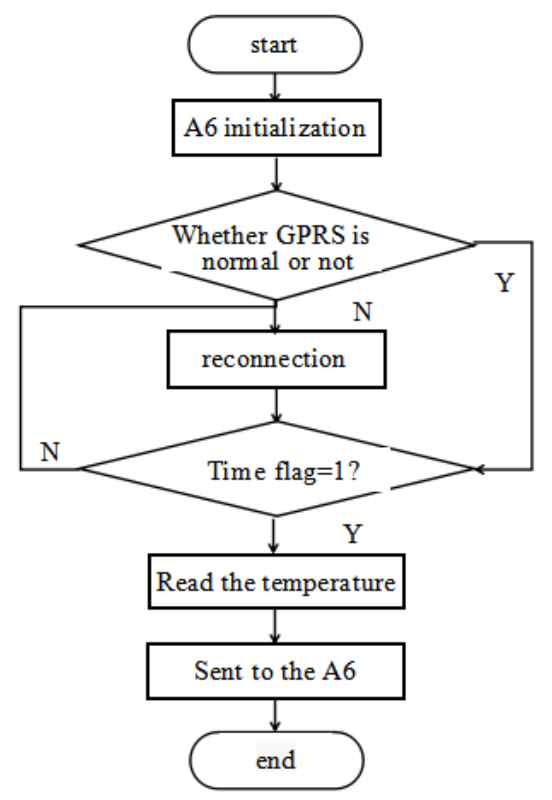

Fig.4 SCM software flow chart

GPRS module software design

Before the system works, the computer should be connected with A6 through the serial port, and the test and input instructions can be carried out through the fruit cloud. After power on module it will be automatically registered network successfully returns + CREG: 1, by sending a TCP message to check up on TCP can send messages, when GPRS of attachment and separation, change the baud rate to 9600 here, get the phone number of the machine and to other phone to dial and send text messages. The detailed instructions for the input will appear below, and of course these instructions are sent according to the standard AT command ${ }^{[6]}$.

Client design

The client (monitoring center) mainly realizes the communication function with the data center (server), and indirectly realizes the measurement and control of the single-chip microcomputer system. The role of TCP/IP in computer communication protocol is to provide a connection-oriented TCP protocol for data communication between the server and the client. At the same time, must pay attention to communicate between the client and the server, to ensure that both use the same communication protocol, both the IP address of the set (including the physical IP address on the server and the client remote IP address), the set port to are the same, 
in addition, when the request of the service, to ensure that the server is in the open state. The software for building a server is network debugging assistant (NetAssist).

Experimental results and analysis

This design mainly studies the transmission of a remote temperature data through GPRS, and can meet the requirements of real-time monitoring and stability. The GPRS module must be mapped out by the computer IP address. If the burning record is not this IP address, it will not be able to transmit data.

Through the experiment on the set up with computer network debugging assistant to collect the test data are sent to, TCP protocol selection TCP Server, for Server's IP address 192.168.1.102, outward open port is 8080, a text box for receiving data, GPRS module is through the address and port to transmit data.

Test on the side and end microcontroller shows the acquisition of temperature,then shows the IP address, and then shows the "SEND OK" that has been set up with computer server is successful, when there is a "SEND SECCESS" co-sponsored by GPRS module has started to SEND data to a computer.

\section{Conclusion}

The system mainly realizes the transmission and reception of temperature data between the microcontroller and the computer, and displays the temperature on the liquid crystal. The paper focuses on the creation of a platform for the communication between the single chip and the computer, and the receiving and sending of temperature data between the end and end. Collect and process temperature data, and communicate with computer through GPRS module. The transmission of GPRS wireless network data provides a powerful means of communication for remote monitoring for some units without wire transfer conditions.

\section{Acknowledgement}

This research is supported by Shandong Provincial Science and Technology Project for Universities under Grant No.J16LN78.

\section{References}

[1]Zhai Weifeng,Zhang Xiaonan,Sun Dehui.Design of environmental monitoring system based on wireless sensor network[J].Journal of agricultural mechanization in China,2016,(06):226-229+242.

[2]Liu Liang, Yan Kewen,Chen Dongdong.Design of remote monitoring system based on GPS and GPRS[J].Industrial control computer,2016,(06):68-69.

[3]Xu Ying.Remote temperature monitoring system based on GPRS[D].Zhejiang:Zhejiang university of technology,2013.

[4]Yang Yifan,Li Bin,Yao Jun,Wang Gaoyang.Design of remote monitoring system based on GPRS[J].Industrial control computer,2016,(12):98-99.

[5]ETSI. General Packet Radio Service (GPRS): Service description, Stage 2 (GSM) 03.60 version 6.4.0 Release 2007.

[6]Douglas E.Comer. Internetworking With TCP/IP Vol I: Principles, Protocols, and Architectures, Publishing House of Electronics Industry, 2011. 